\title{
Mermin inequalities for perfect correlations
}

\author{
Adán Cabello, , , Otfried Gühne, ${ }^{2,3}$ and David Rodríguez ${ }^{4}$ \\ ${ }^{1}$ Departamento de Física Aplicada II, Universidad de Sevilla, E-41012 Sevilla, Spain \\ ${ }^{2}$ Institut für Quantenoptik und Quanteninformation, \\ Österreichische Akademie der Wissenschaften, A-6020 Innsbruck, Austria \\ ${ }^{3}$ Institut für Theoretische Physik, Universität Innsbruck, A-6020 Innsbruck, Austria \\ ${ }^{4}$ Departamento de Física Aplicada III, Universidad de Sevilla, E-41092 Sevilla, Spain
}

(Dated: October 24, 2018)

\begin{abstract}
Any $n$-qubit state with $n$ independent perfect correlations is equivalent to a graph state. We present the optimal Bell inequalities for perfect correlations and maximal violation for all classes of graph states with $n \leq 6$ qubits. Twelve of them were previously unknown and four give the same violation as the Greenberger-Horne-Zeilinger state, although the corresponding states are more resistant to decoherence.

PACS numbers: 03.65.Ud, 03.67.Mn, 03.67.Pp, 42.50.-p
\end{abstract}

\section{INTRODUCTION}

In 1989, Greenberger, Horne, and Zeilinger (GHZ) showed that no local hidden variable (LHV) theory can assign predefined local results which agree with the perfect correlations predicted by quantum mechanics for separated measurements on $n \geq 3$ distant sites on a system prepared in the $n$-qubit GHZ state [1]. Mermin converted the $n$-party GHZ proof into a violation of a $n$-party Bell inequality [2]. The amount of the violation of Mermin's inequalities, measured by the ratio $\mathcal{D}$ between the quantum value of the Bell operator and its bound in LHV theories, grows exponentially with $n$. For a given $n$ (with $n$ odd), Mermin's inequality gives the maximal possible violation of any $n$-party two-setting Bell inequality in quantum mechanics [3].

Can we extend this result to other $n$-qubit states? The essential ingredient for GHZ-type proofs and Mermintype Bell inequalities is that they require an $n$-qubit quantum state, which is a simultaneous eigenstate of $n$ commuting local observables (i.e., a stabilizer state). Any stabilizer state is, up to local rotations, equivalent to a graph state [4] (i.e., a stabilizer state whose generators can be written with the help of a graph [5]). These states are essential in quantum error correction [6] and one-way quantum computation 7]. For a small number of qubits, all classes of nonequivalent graph states under single-qubit unitary transformations are known [5]. For $3 \leq n \leq 6$, there are thirteen different classes of graph states which are nonequivalent to GHZ states. For a given $n$, some of them are more robust against decoherence than the GHZ state [8].

Bell inequalities for graph states constitute a subject of intense study $9,10,11,12,13,14,15,16]$. However, the Mermin inequalities for most of them are unknown. For a given state, the Mermin inequality is the Bell inequality such that (I) the Bell operator is a sum of stabilizing

*Electronic address: adan@us.es operators of that state (i.e., perfect correlations), and (II) the violation is maximal. If the maximum is obtained for Bell operators with a different number of terms, then we will choose the one with the lowest number, since the other inequalities contain this inequality and require more measurements. For some graph states, the Mermin inequality is not unique due to the symmetries of the graph.

This definition is motivated by the relation between the original GHZ proof [1] and the Mermin inequality 2]. The aim of this paper is to introduce the Mermin inequalities for all graph states (or, equivalently, for all pure states with $n$ independent perfect correlations) with $n<7$ qubits.

The graph state $|G\rangle$ is the unique $n$-qubit state that satisfies $g_{i}|G\rangle=|G\rangle$, for $i=1, \ldots, n$, where $g_{i}$ are the generators of the stabilizer group of the state, defined as the set $\left\{s_{j}\right\}_{j=1}^{2^{n}}$ of all products of the generators. The perfect correlations of the graph state are

$$
\left\langle G\left|s_{j}\right| G\right\rangle=1 \text { for } j=1, \ldots, 2^{n} .
$$

The $g_{i}$ 's are obtained with the help of a graph $G$. For instance, the $n$-qubit GHZ state is associated to the starshaped graph in which qubit 1 is connected to all the other qubits. This means that $g_{1}=X_{1} \bigotimes_{i \neq 1}^{n} Z_{i}$ and $g_{i}=X_{1} \otimes Z_{i}$ for $i \neq 1 ; X_{i}, Y_{i}$, and $Z_{i}$ denote the Pauli matrices acting on the $i$ th qubit (see [5] for more details).

There are many possible GHZ-like proofs for a given graph state associated to a connected graph of $n \geq 3$ qubits. All of them have the same structure. Any LHV theory assigning predefined values -1 or 1 to $X_{i}, Y_{i}$, and $Z_{i}$ in agreement with the quantum predictions given by (11) must satisfy

$$
s_{j}=1 \text { for } j=1, \ldots, 2^{n} .
$$

However, if we choose a suitable subset of $q$ predictions from the set (2), and assume predefined values, either -1 or 1 , then for some choices it happens that, at most, only $p<q$ of these predictions are satisfied. For the remaining $q-p$ quantum predictions, the prediction of 
the LHV theory is the opposite: $s_{j}=-1$. This difference can be reformulated as a violation of the Bell inequality

$$
\beta \leq 2 p-q,
$$

where the Bell operator $\beta$ is the sum of the stabilizing operators of the chosen subset. According to Eq. (10), the graph state satisfies

$$
\langle G|\beta| G\rangle=q .
$$

Therefore, $|G\rangle$ violates the inequality (3) by an amount $\mathcal{D}=q /(2 p-q)$. For the GHZ proof with $n$ odd, the maximum contradiction, measured by $q / p$, and the maximum violation of the Bell inequality, measured by $\mathcal{D}$, is obtained when $q=2^{n-1}$ and $p=2^{n-2}+2^{(n-3) / 2}$. This is Mermin's inequality [2]. If we take a different subset of stabilizing operators, then we can have a violation of a Bell inequality, but usually not the maximum one.

Specifically, for a given graph state associated to a connected graph of $n \geq 3$ qubits, if we consider the Bell operator consisting of the whole set of stabilizing operators, then we always have a violation of a Bell inequality [10], but not the maximum one. A violation occurs because that Bell operator contains a simpler Bell operator giving the maximum violation.

Why are we interested in those Bell inequalities with the maximum $\mathcal{D}$ ? $\mathcal{D}$ is the measure of nonlocality used in Refs. [2, 3, 10]. For graph states and stabilizer Bell inequalities, it is well defined, easily computable, and the two practical measures of nonlocality, the resistance to noise and the detection efficiency for a loophole-free Bell experiment, are connected to $\mathcal{D}$.

(i) In actual experiments, instead of a pure state $|G\rangle$, we usually have a noisy one, $\rho=\mathcal{V}|G\rangle\langle G|+(1-\mathcal{V}) \mathbb{1} / 2^{n}$, where $\mathbb{1}$ is the identity matrix in the Hilbert space of the whole system. $\mathcal{D}$ is related to the minimum value of $\mathcal{V}$ required to actually observe a violation of the Bell inequality $\mathcal{V}_{\text {crit }}$. For graph states and stabilizer Bell inequalities, if $\mathcal{D}$ increases, then $\mathcal{V}_{\text {crit }}$ decreases. Specifically, a simple calculation gives that $\mathcal{V}_{\text {crit }}=1 / \mathcal{D}$.

(ii) An open problem in fundamental physics is achieving a loophole-free Bell experiment. A particularly important problem is the detection loophole [17]. $\mathcal{D}$ is related to the minimum detection efficiency required for a loophole-free Bell experiment $\eta_{\text {crit }}$. For graph states and stabilizer Bell inequalities, if $\mathcal{D}$ increases, then $\eta_{\text {crit }}$ decreases. Specifically, for GHZ states and the Mermin inequality with $n$ odd, $\eta_{\text {crit }}=[2+(\log 2 / \log \mathcal{D})] / 4[18]$.

(iii) In addition, $\mathcal{D}$ provides the relevant parameters of the underlying GHZ-type proof: $q$ and $p$. Any GHZ-type proof can be converted into an $n$-party quantum pseudotelepathy game in which a team assisted with a graph state always wins, while a team with only classical resources wins only with probability $p / q[19]$. Therefore, the higher $\mathcal{D}$, the lower $p / q$ and the higher quantum advantage.

The knowledge of the Mermin inequalities for all graph states is important for (a) Quantum information. Graph states are essential for quantum information tasks. Mermin inequalities are useful tools for their experimental analysis. For instance, in recent experiments preparing 6-qubit graph states $\mathcal{V}$ is around 0.5 20, 21, 22], thus Bell inequalities with $\mathcal{D}>2$ are required to observe violation. We will show that for all 6-qubit graph states, Mermin inequalities have $\mathcal{D}>2$.

(b) Nonlocality vs decoherence experiments. For GHZ states, $\mathcal{D}$ increases exponentially with $n$ [2]. However, GHZ states' entanglement lifetime under decoherence decreases with $n$ [8]. Therefore, a fundamental limitation seemingly exists to observe macroscopic violations of Bell inequalities with GHZ states. A natural question is: Does this limitation also hold for other types of graph states? What happens to those graph states whose lifetime under decoherence does not decrease with $n[8]$ ? To answer these questions we need to know how $\mathcal{D}$ scales with $n$ within a family of graph states, and which graph states have higher $\mathcal{D}$.

\section{MERMIN INEQUALITIES FOR GRAPH STATES}

For each graph state, our task is to obtain, from all possible Bell operators which are sums of stabilizing operators, those which provide the highest violation. The exhaustive study for $n \geq 6$ becomes computationally difficult because the number of potential Bell operators to test scales like $2^{2^{n}}$. However, if we restrict our attention to Bell operators with the same symmetry as the underlying graph, this investigation is still computationally feasible for $n=6$.

In Table \ we present all the Mermin inequalities for all graph states with $2<n<6$ qubits. In Table I we present the Mermin inequalities possessing the same symmetry as the underlying graph for all graph states with $n=6$ qubits. In both tables we have followed the classification and the labeling of the qubits of Fig. 1 (taken from Ref. [5]). $\mathrm{LC}_{n}\left(\mathrm{RC}_{n}\right)$ denotes the $n$-qubit linear (ring) cluster state [10], $Y_{5}$ denotes the 5-qubit graph state associated to the graph " $Y$ ", $H_{6}$ the 6 -qubit graph state associated to the graph " $H$ ", etc. The quantum prediction for each Bell operator $\beta$ is $q$ (i.e., the number of terms of $\beta$ ); $p$ is the maximum number of the $q$ perfect correlations that a LHV theory can satisfy; $\mathcal{D}=\frac{q}{2 p-q}$ is the violation of the Bell inequality $\beta \leq 2 p-q$.

Some of the inequalities in Tables [ and [1] were previously known. For the $n$-qubit GHZ states with $n$ odd, we recover the original Mermin inequalities [2]. For the $n$-qubit GHZ states with $n$ even, the original Mermin inequalities are the sum of our two symmetric inequalities (the fist two inequalities for the $\mathrm{GHZ}_{4}$ in Table $\square$ and the two inequalities for the $\mathrm{GHZ}_{6}$ in Table (I). Our inequalities have the same violation as Mermin's, but only half of the terms. For $n$ even, Ardehali proposed a method giving an additional violation of $\sqrt{2}$ [23]. Ardehali's inequalities do not use only perfect correlations. Ardehali's 
TABLE I: Mermin inequalities for all graph states of $n<6$ qubits.

\begin{tabular}{|c|c|c|c|c|}
\hline Graph state & $g_{i}$ & $\beta \leq 2 p-q$ & Settings & $\mathcal{D}$ \\
\hline $2\left(\mathrm{GHZ}_{3}\right)$ & $\begin{array}{c}g_{1}=X_{1} Z_{2} Z_{3} \\
g_{i}=Z_{1} X_{i} \text { for } i \neq 1\end{array}$ & $g_{1}\left(\mathbb{1}+g_{2}\right)\left(\mathbb{1}+g_{3}\right) \leq 2$ & $2-2-2$ & 2 \\
\hline $3\left(\mathrm{GHZ}_{4}\right)$ & $\begin{array}{c}g_{1}=X_{1} Z_{2} Z_{3} Z_{4} \\
g_{i}=Z_{1} X_{i} \text { for } i \neq 1\end{array}$ & $\begin{array}{c}g_{1}\left(\mathbb{1}+g_{2} g_{3}+g_{2} g_{4}+g_{3} g_{4}\right) \leq 2 \text { and } g_{1} \rightarrow g_{1} g_{2} \\
g_{1}\left(\mathbb{1}+g_{i}\right)\left(\mathbb{1}+g_{j}\right) \leq 2 \text { and } g_{1} \rightarrow g_{1} g_{k}\end{array}$ & $\begin{array}{c}1-2-2-2 \\
2-2(i)-2(j)-1(k)\end{array}$ & 2 \\
\hline $4\left(\mathrm{LC}_{4}\right)$ & $\begin{array}{c}g_{1}=X_{1} Z_{2}, g_{4}=Z_{3} X_{4} \\
g_{i}=Z_{i-1} X_{i} Z_{i+1} \text { for } i=2,3\end{array}$ & $\begin{array}{c}\left(\mathbb{1}+g_{1}\right) g_{2}\left(\mathbb{1}+g_{3}\right) \leq 2 \text { and } g_{3} \rightarrow g_{3} g_{4} \\
\left(\mathbb{1}+g_{1}\right) g_{2}\left(g_{3}+g_{4}\right) \leq 2 \text { and } g_{3} \rightarrow g_{3} g_{4} \\
g_{i} \rightarrow g_{i+1}\end{array}$ & $\begin{array}{l}2-2-2-1 \\
2-2-1-2\end{array}$ & 2 \\
\hline $5\left(\mathrm{GHZ}_{5}\right)$ & $\begin{array}{l}g_{1}=X_{1} Z_{2} Z_{3} Z_{4} Z_{5} \\
g_{i}=Z_{1} X_{i} \text { for } i \neq 1\end{array}$ & $g_{1}\left(\mathbb{1}+g_{2}\right)\left(\mathbb{1}+g_{3}\right)\left(\mathbb{1}+g_{4}\right)\left(\mathbb{1}+g_{5}\right) \leq 4$ & $2-2-2-2-2$ & 4 \\
\hline $6\left(Y_{5}\right)$ & $\begin{array}{c}g_{1}=X_{1} Z_{2}, g_{5}=Z_{2} X_{5} \\
g_{2}=Z_{1} X_{2} Z_{5} \\
g_{3}=Z_{2} X_{3} Z_{4} \\
g_{4}=Z_{3} X_{4}\end{array}$ & $\begin{array}{c}g_{2}\left[\left(\mathbb{1}+g_{1}+g_{5}\right)\left(\mathbb{1}+g_{3}+g_{3} g_{4}\right)+\left(\mathbb{1}+g_{1} g_{5}\right) g_{4}\right] \\
+\left(g_{1}+g_{5}\right) g_{3}\left(\mathbb{1}+g_{4}\right) \leq 7 \\
g_{2} \rightarrow g_{2} g_{4} \\
\beta \rightarrow g_{4} \beta \text { and } 32 \text { nonsymmetric more }\end{array}$ & $\begin{array}{l}3-3-3-3-2 \\
3-3-3-3-3\end{array}$ & $\frac{15}{7}$ \\
\hline $7\left(\mathrm{LC}_{5}\right)$ & $\begin{array}{c}g_{1}=X_{1} Z_{2}, g_{5}=Z_{4} X_{5} \\
g_{i}=Z_{i-1} X_{i} Z_{i+1} \text { for } i=2,3,4\end{array}$ & $\left(\mathbb{1}+g_{1}\right)\left[\left(\mathbb{1}+g_{2}\right) g_{3}\left(\mathbb{1}+g_{4}\right)+g_{2} g_{4}\right]\left(\mathbb{1}+g_{5}\right) \leq 8$ & $3-3-3-3-3$ & $\frac{5}{2}$ \\
\hline \multirow[t]{2}{*}{$8\left(\mathrm{RC}_{5}\right)$} & $g_{i}=Z_{i-1} X_{i} Z_{i+1}$ & $\gamma+\sum_{i=1}^{5} g_{i} g_{i+1} \leq 9$ & $3-3-3-3-3$ & $\frac{7}{3}$ \\
\hline & & $\begin{array}{c}\gamma+g_{j} g_{i+1}+g_{i} g_{i+2}+g_{i-1} g_{i+1} \\
+g_{i-2} g_{i} g_{i+1} g_{i+2}+g_{i-2} g_{i-1} g_{i} g_{i+1} \leq 9 \\
\gamma=\frac{1}{2}\left[\prod_{i=1}^{5}\left(\mathbb{1}+g_{i}\right)-\prod_{i=1}^{5}\left(\mathbb{1}-g_{i}\right)\right] \\
\text { and } 105 \text { more }\end{array}$ & $3-3-3-3-3$ & \\
\hline
\end{tabular}

method can be extended to other graph states [24]. Mermin's inequalities have been tested in the laboratory using 3- 25] and 4-qubit GHZ states [26, 27]. Sources of 5[28] and 6-qubit GHZ states [20, 21] already exist.

For the 4-qubit cluster state $\left(\mathrm{LC}_{4}\right)$, the Mermin inequalities in Table \ contain those introduced in [11, 12]. These inequalities have been recently tested in the laboratory [29, 30, 31].

However, twelve of the Mermin inequalities in Tables 1 and II are new. They include those for the $\mathrm{RC}_{5}$, important for quantum error correction codes [6] ; for the $\mathrm{H}_{6}$, a universal resource for one-way quantum computation recently prepared in the laboratory [21]; and for the $\mathrm{Y}_{6}$, which allows a demonstration of anyonic statistics in the Kitaev model 22, 32, 33.

A remarkable fact is, that four 6-qubit graph states have the same violation as the $\mathrm{GHZ}_{6}$ : the graph state no. 10 , the $H_{6}$, the $Y_{6}$, and the $\mathrm{LC}_{6}$ (see Table $\amalg$ ). This is interesting because these states are more resistant to decoherence than the $\mathrm{GHZ}_{6}[8]$. This proves that the nonlocality vs decoherence ratio of GHZ states is not universal: there are states with similar violations but that are more robust against decoherence.

\section{Acknowledgments}

The authors thank H. J. Briegel, P. Moreno, and G. Vallone for useful discussions. A.C. acknowledges support from the Spanish MEC Project No. FIS2005-07689, and the Junta de Andalucía Excellence Project No. P06FQM-02243. O.G. acknowledges support from the FWF and the EU (OLAQUI, SCALA, and QICS).
[1] D. M. Greenberger, M. A. Horne, and A. Zeilinger, in Bell's Theorem, Quantum Theory, and Conceptions of the Universe, edited by M. Kafatos (Kluwer Academic, Dordrecht, Holland, 1989), p. 69.

[2] N. D. Mermin, Phys. Rev. Lett. 65, 1838 (1990).

[3] R. F. Werner and M. M. Wolf, Phys. Rev. A 64, 032112 (2001).

[4] M. Van den Nest, J. Dehaene, and B. De Moor, Phys. Rev. A 69, 022316 (2004).

[5] M. Hein, J. Eisert, and H. J. Briegel, Phys. Rev. A 69,
062311 (2004).

[6] D. Gottesman, Phys. Rev. A 54, 1862 (1996).

[7] R. Raussendorf and H. J. Briegel, Phys. Rev. Lett. 86, 5188 (2001).

[8] W. Dür and H. J. Briegel, Phys. Rev. Lett. 92, 180403 (2004).

[9] D. P. DiVincenzo and A. Peres, Phys. Rev. A 55, 4089 (1997).

[10] O. Gühne, G. Tóth, P. Hyllus, and H. J. Briegel, Phys. Rev. Lett. 95, 120405 (2005). 
TABLE II: Symmetric Mermin inequalities for all graph states of $n=6$ qubits.

\begin{tabular}{|c|c|c|c|c|}
\hline Graph state & $g_{i}$ & $\beta \leq 2 p-q$ & Settings & $\mathcal{D}$ \\
\hline \multirow[t]{2}{*}{$9\left(\mathrm{GHZ}_{6}\right)$} & $g_{1}=X_{1} Z_{2} Z_{3} Z_{4} Z_{5} Z_{6}$ & $g_{1}\left(\mathbb{1}+\sum_{i \neq j \neq 1} g_{i} g_{j}+\sum_{i \neq j \neq k \neq l \neq 1} g_{i} g_{j} g_{k} g_{l}\right) \leq 4$ & $1-2-2-2-2-2$ & 4 \\
\hline & $g_{i}=Z_{1} X_{i}$ for $i \neq 1$ & and $g_{1} \rightarrow g_{1} g_{2}$ & $1-2-2-2-2-2$ & \\
\hline \multirow{2}{*}{10} & $g_{4}=X_{4} Z_{5}$ & $g_{5} \rightarrow g_{4} g_{5}$ & $2-2-2-1-2-2$ & \\
\hline & $g_{5}=Z_{4} X_{5} Z_{6}$ & $\left(\mathbb{1}+g_{1}\right)\left(\mathbb{1}+g_{2}\right)\left(\mathbb{1}+g_{3}\right)\left(g_{4}+g_{5}\right) g_{6} \leq 4$ & $2-2-2-2-1-2$ & \\
\hline \multirow{3}{*}{$11\left(\mathrm{H}_{6}\right)$} & $g_{3}=X_{3} Z_{5}, g_{4}=X_{4} Z_{5}$ & $g_{1} \leftrightarrow g_{2}$ (i.e., permute them) & $2-1-3-3-3-2$ & \\
\hline & $g_{5}=Z_{3} Z_{4} X_{5}, g_{6}=Z_{1} Z_{2} X_{6}$ & $\left(\mathbb{1}+g_{1}\right)\left(\mathbb{1}+g_{2}\right) g_{3}\left(\mathbb{1}+g_{4}\right) g_{5}\left(\mathbb{1}+g_{6}\right) \leq 4$ & $3-3-1-2-2-3$ & \\
\hline & & $g_{3} \leftrightarrow g_{4}$ & $3-3-2-1-2-3$ & \\
\hline $12\left(\mathrm{Y}_{6}\right)$ & $\begin{array}{c}g_{1}=X_{1} Z_{2}, g_{6}=Z_{2} X_{6} \\
g_{2}=Z_{1} X_{2} Z_{3} Z_{6}, g_{3}=Z_{2} X_{3} Z_{4} \\
g_{4}=Z_{3} X_{4} Z_{5}, g_{5}=Z_{4} X_{5}\end{array}$ & $\left(\mathbb{1}+g_{1}\right) g_{2}\left(\mathbb{1}+g_{3}\right) g_{4}\left(\mathbb{1}+g_{5}\right)\left(\mathbb{1}+g_{6}\right) \leq 4$ & $2-2-1-2-2-2$ & 4 \\
\hline $13\left(\mathrm{E}_{6}\right)$ & $g_{3}=Z_{2} X_{3} Z_{4} Z_{6}, g_{6}=Z_{3} X_{6}$ & and 37 more & & \\
\hline $14\left(\mathrm{LC}_{6}\right)$ & $\begin{array}{c}g_{1}=X_{1} Z_{2}, g_{6}=Z_{5} X_{6} \\
g_{i}=Z_{i-1} X_{i} Z_{i+1} \text { for } i=2,3,4,5\end{array}$ & $\left(\mathbb{1}+g_{1}\right) g_{2}\left(\mathbb{1}+g_{3}\right)\left(\mathbb{1}+g_{4}\right) g_{5}\left(\mathbb{1}+g_{6}\right) \leq 4$ & $2-2-3-3-2-2$ & 4 \\
\hline \multirow[t]{2}{*}{15} & $g_{1}=X_{1} Z_{6}, g_{2}=X_{2} Z_{4}$ & $\left(g_{3}+g_{5}\right)\left(\mathbb{1}+g_{1}\right)\left(\mathbb{1}+g_{2}\right)\left(\mathbb{1}+g_{4}\right)\left(\mathbb{1}+g_{6}\right)$ & & \\
\hline & $\begin{array}{c}g_{3}=X_{3} Z_{4} Z_{6}, g_{5}=Z_{4} X_{5} Z_{6} \\
g_{4}=Z_{2} Z_{3} X_{4} Z_{5}, g_{6}=Z_{1} Z_{3} Z_{5} X_{6}\end{array}$ & $\begin{array}{c}+\left(\mathbb{1}+g_{3} g_{5}\right)\left(g_{4}+g_{2} g_{4}+g_{6}+g_{1} g_{6}\right) \leq 16 \\
\text { and } 6 \text { more }\end{array}$ & $3-3-3-3-3-3$ & $\frac{5}{2}$ \\
\hline \multirow[t]{3}{*}{16} & $g_{1}=X_{1} Z_{2}, g_{5}=Z_{4} X_{5}$ & $g_{3}\left(\mathbb{1}+g_{1}+g_{2}+g_{1} g_{2}+g_{4}+g_{5}+g_{4} g_{5}\right)\left(\mathbb{1}+g_{6}\right)$ & & \\
\hline & $g_{2}=Z_{1} X_{2} Z_{3} Z_{4}, g_{4}=Z_{2} Z_{3} X_{4} Z_{5}$ & $+\left(\mathbb{1}+g_{1}\right) g_{2}\left(\mathbb{1}+g_{5}+g_{6}\right)+g_{4}\left(\mathbb{1}+g_{5}\right)\left(\mathbb{1}+g_{1}+g_{6}\right)$ & & \\
\hline & $\begin{array}{c}g_{3}=Z_{2} X_{3} Z_{4} Z_{6} \\
g_{6}=Z_{3} X_{6}\end{array}$ & $\begin{array}{c}+\left(\mathbb{1}+g_{1}\right) g_{2} g_{4}\left(\mathbb{1}+g_{5}\right) \leq 12 \\
\text { and } 3 \text { more }\end{array}$ & $3-3-3-3-3-3$ & 3 \\
\hline \multirow{2}{*}{19} & $g_{2}=Z_{1} X_{2} Z_{3} Z_{5}, g_{5}=Z_{2} Z_{4} X_{5} Z_{6}$ & $+g_{5}\left(g_{1}+g_{3}+g_{1} g_{3}\right)+\left(g_{2}+g_{5}\right)\left(g_{3} g_{4}+g_{1} g_{6}+g_{1} g_{3} g_{4} g_{6}\right)$ & & \\
\hline & $g_{3}=Z_{1} Z_{2} X_{3} Z_{4}, g_{6}=Z_{1} Z_{4} Z_{5} X_{6}$ & $+g_{2} g_{5}\left[g_{1} g_{4}\left(\mathbb{1}+g_{3}+g_{6}\right)+g_{3} g_{6}\left(\mathbb{1}+g_{1}+g_{4}\right)\right] \leq 9$ & $3-3-3-3-3-3$ & $\frac{7}{3}$ \\
\hline
\end{tabular}

[11] A. Cabello, Phys. Rev. Lett. 95, 210401 (2005).

[12] V. Scarani, A. Acín, E. Schenck, and M. Aspelmeyer, Phys. Rev. A 71, 042325 (2005).

[13] G. Tóth, O. Gühne, and H. J. Briegel, Phys. Rev. A 73, 022303 (2006).

[14] L.-Y. Hsu, Phys. Rev. A 73, 042308 (2006).

[15] J. Barrett, C. M. Caves, B. Eastin, M. B. Elliott, and S. Pironio, Phys. Rev. A 75, 012103 (2007).

[16] C. Wu, Y. Yeo, L. C. Kwek, and C. H. Oh, Phys. Rev. A 75, 032332 (2007).

[17] P. M. Pearle, Phys. Rev. D 2, 1418 (1970).

[18] A. Cabello, D. Rodríguez, and I. Villanueva e-print arXiv:0712.3268.

[19] A. Cabello, Phys. Rev. A 73, 022302 (2006).

[20] D. Leibfried, E. Knill, S. Seidelin, J. Britton, R. B. Blakestad, J. Chiaverini, D. B. Hume, W. M. Itano, J. D. Jost, C. Langer, R. Ozeri, R. Reichle, and D. J.
Wineland, Nature (London) 438, 639 (2005).

[21] C.-Y. Lu, X.-Q. Zhou, O. Gühne, W.-B. Gao, J. Zhang, Z.-S. Yuan, A. Goebel, T. Yang, and J.-W. Pan, Nat. Phys. 3, 91 (2007).

[22] C.-Y. Lu, W.-B. Gao, O. Gühne, X.-Q. Zhou, Z.-B. Chen, and J.-W. Pan, e-print arXiv:0710.0278.

[23] M. Ardehali, Phys. Rev. A 46, 5375 (1992).

[24] O. Gühne and A. Cabello, Phys. Rev. A 77, 032108 (2008).

[25] J.-W. Pan, D. Bouwmeester, M. Daniell, H. Weinfurter, and A. Zeilinger, Nature (London) 403, 515 (2000).

[26] C. A. Sackett, D. Kielpinski, B. E. King, C. Langer, V. Meyer, C. J. Myatt, M. Rowe, Q. A. Turchette, W. M. Itano, D. J. Wineland, and C. Monroe, Nature (London) 404, 256 (2000).

[27] Z. Zhao, T. Yang, Y.-A. Chen, A.-N. Zhang, M. Żukowski, and J.-W. Pan, Phys. Rev. Lett. 91, 180401 


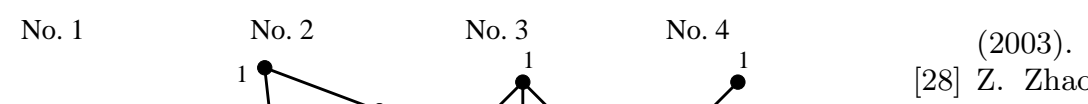

[28] Z. Zhao, Y.-A. Chen, A.-N. Zhang, T. Yang, H. J. Briegel, and J.-W. Pan, Nature (London) 430, 54 (2004).

[29] P. Walther, M. Aspelmeyer, K. J. Resch, and A. Zeilinger, Phys. Rev. Lett. 95, 020403 (2005).

[30] N. Kiesel, C. Schmid, U. Weber, G. Tóth, O. Gühne, R. Ursin, and H. Weinfurter, Phys. Rev. Lett. 95, 210502 (2005).
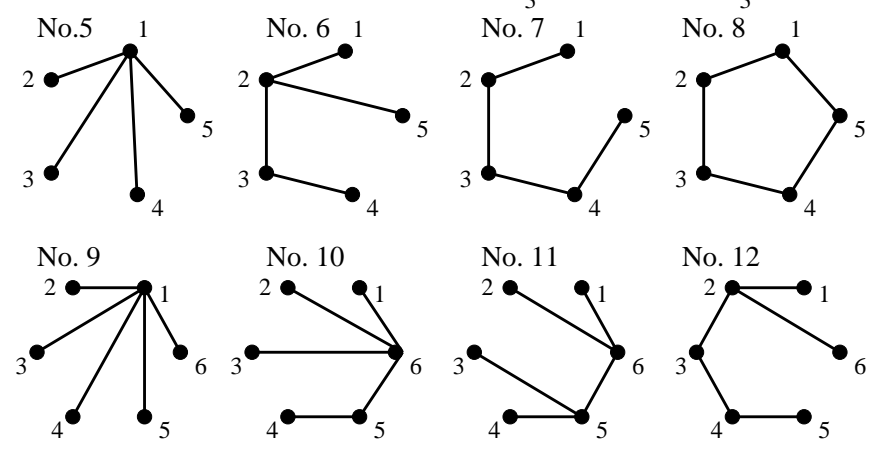

[31] G. Vallone, E. Pomarico, P. Mataloni, F. De Martini, and V. Berardi, Phys. Rev. Lett. 98, 180502 (2007).

[32] Y.-J. Han, R. Raussendorf, and L.-M. Duan, Phys. Rev. Lett. 98, 150404 (2007).

[33] J. K. Pachos, W. Wieczorek, C. Schmid, N. Kiesel, R. Pohlner, and H. Weinfurter, e-print arXiv:0710.0895.
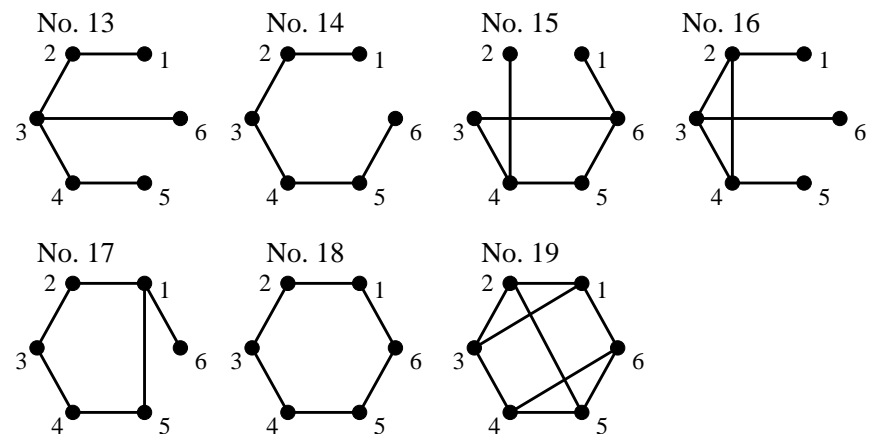

FIG. 1: Graphs representing all classes of $n$-qubit graph states, with $2 \leq n \leq 6$, that are inequivalent under singlequbit unitary transformations and graph isomorphism. The figure is taken from Ref. [5]. 